\title{
A AQUISIÇÃO DE ALIMENTOS PARA A ALIMENTAÇÃO ESCOLAR EM MARAPANIM-PA
}

\section{Tahnity Haarad Moura CHAVES $^{1}$; Rosana Quaresma MANESCHY2; Wagner Luiz Ramos BARBOSA $^{3}$.}

\author{
${ }^{1}$ Mestranda do PPGEDAM, Núcleo de Meio Ambiente (NUMA) da Universidade Federal do \\ Pará (UFPA), Belém, Pará, Brasil, tahnityhaarad@gmail.com \\ 2PPGEDAM/NUMA/UFPA, Belém, Pará, Brasil romaneschy@ufpa.br \\ 2PPGEDAM/Instituto de Ciências da Saúde/UFPA, Belém, Pará, Brasil, barbosa@ufpa.br
}

\begin{abstract}
RESUMO: As políticas públicas federais para a Agricultura Familiar (AF) têm sido importantes na promoção do desenvolvimento local, sobretudo na Amazônia. Dentre as políticas de aquisição de alimentos estão as ações de Segurança Alimentar Nacional para que as pessoas tenham acesso a alimentação adequada. O Programa Nacional de Alimentação Escolar (PNAE) é um programa do Governo Federal que tem o objetivo de fornecer alimentos para a merenda escolar a partir da produção de AF. Essa pesquisa teve como objetivo levantar os principais entraves a aquisição de alimentos para a alimentação escolar no município de Marapanim-PA via PNAE. A abordagem da pesquisa foi qualitativa e utilizou-se a metodologia de estudo de caso para análise do material coletado a partir de pesquisa bibliográfica, documental, observação a partir de visitas in loco e entrevistas com informantes-chave. Verificou-se que o município vem recebendo os repasses do PNAE, no entanto não houve fornecimento de merenda escolar de março a maio de 2017. O município possui produção agropecuária diversificada com potencial para atender a demanda do PNAE, porém não existe uma sistematização dos dados da produção agropecuária no município para orientar programas e ações municipais. A gestão do município não atua de forma intersetorial e nem promove ações participativas para envolver os sujeitos com potencial interesse no PNAE.
\end{abstract}

PALAVRAS-CHAVE: Agricultura familiar, Desenvolvimento local, Políticas públicas.

\section{THE ACQUISITION OF FOODS FOR SCHOOL FEEDING IN MARAPANIM-PA}

ABSTRACT: Federal public policies for Family Agriculture (FA) have been important in promoting local development, especially in the Amazon. Among the food acquisition policies are the National Food Safety actions so that people have access to adequate food. The National School Feeding Program (NSFP) is a Federal Government program that aims to provide food for school meals from the production of FA. This research had as objective to raise the main obstacles to the acquisition of food for school feeding in the municipality of Marapanim-PA via NSFP. The research approach was qualitative and the case study methodology was used to analyze the material collected from bibliographical research, 
documentary, observation from on-site visits and interviews with key informants. It was verified that the municipality has been receiving the NSFP onlendings, however there was no supply of school meals from March to May 2017. The municipality has diversified agricultural production with potential to meet the NSFP demand, but there is no data systematization of agricultural production in the municipality to guide municipal programs and actions. The municipality's management does not act in an intersectoral way nor does it promote participatory actions to involve the subjects with potential interest in the NSFP.

KEYWORDS: Family agriculture, Local development, Public policies.

\section{LA ADQUISICIÓN DE ALIMENTOS PARA LA ALIMENTACIÓN ESCOLAR EN MARAPANIM-PA}

RESUMEN: Las políticas públicas federales para la Agricultura Familiar (AF) han sido importantes en la promoción del desarrollo local, sobre todo en la Amazonia. Entre las políticas de adquisición de alimentos están las acciones de Seguridad Alimentaria Nacional para que las personas tengan acceso a la alimentación adecuada. El Programa Nacional de Alimentación Escolar (PNAE) es un programa del Gobierno Federal que tiene el objetivo de suministrar alimentos para la merienda escolar a partir de la producción de AF. Esta investigación tuvo como objetivo levantar los principales obstáculos a la adquisición de alimentos para la alimentación escolar en el municipio de Marapanim-PA vía PNAE. El enfoque de la investigación fue cualitativo y se utilizó la metodología de estudio de caso para análisis del material recogido a partir de la investigación bibliográfica, documental, observación a partir de visitas in situ y entrevistas con informantes clave. Se verificó que el municipio viene recibiendo los traspasos del PNAE, sin embargo, no hubo suministro de merienda escolar de marzo a mayo de 2017. El municipio posee producción agropecuaria diversificada con potencial para atender la demanda del PNAE, pero no existe una sistematización de los datos de la producción agropecuaria en el municipio para orientar programas y acciones municipales. La gestión del municipio no actúa de forma intersectorial y no promueve acciones participativas para involucrar a los sujetos con potencial interés en el PNAE.

PALABRAS CLAVE: Agricultura familiar, Desarrollo local, Políticas públicas.

A partir dos anos 1990 foram implantadas diversas políticas públicas demandadas pela categoria produtiva "agricultura familiar" (AF). Essas políticas têm sido importantes na promoção de desenvolvimento local a partir da formulação e implementação de ações municipais de Segurança Alimentar Nacional (SAN) para atender ao Direito Humano à Alimentação Adequada 
(DHAA). Nesse contexto, a aquisição de alimentos via mercado institucional, como - Programa Nacional de Alimentação Escolar (PNAE) tem incentivando o consumo de produtos da agricultura familiar local.

As políticas públicas visam à melhoria de vida dos agricultores, que além dos aspectos produtivos, tem se voltado para o uso de práticas agroecológicas e à conservação dos recursos naturais (GAVIOLI, 2001).

Segundo BRASIL (2017), em 2017 a compra institucional de alimentos provenientes da agricultura familiar no Brasil deve crescer 430\%. O PNAE é a política pública mais antiga do país e fornece alimentos para a merenda de escolas e creches em caráter universal, "promovendo a alimentação adequada e saudável como ferramenta para a Segurança Alimentar e Nutricional" (MINISTÉRIO DO DESENVOLVIMETO SOCIAL E AGRÁRIO, 2017).

A Lei Federal n¹1.947/09 "estabelece que pelo menos 30\% dos recursos dos programas de aquisição de alimentos sejam aplicados na compra de produtos da agricultura familiar". No âmbito municipal, verifica-se que na região norte o Programa de Aquisição de alimentos (PAA) e o PNAE tem sido um incentivo à produção agrícola, a geração de renda e ao desenvolvimento local (HONDA et al. 2016; SANTANA et al., 2017; SCHRÖDE, 2010). Porém, existem municípios com dificuldades em utilizar esse recurso, que vão desde entraves referentes a falta de políticas intersetoriais na gestão municipal até a participação da sociedade civil no processo (BRAGA; AZEVEDO, 2012). Essa pesquisa teve como objetivo levantar os principais entraves a aquisição de alimentos para a alimentação escolar no município de Marapanim-PA via PNAE.

A pesquisa se desenvolveu a partir de um estudo de caso, que teve como lócus a categoria produtiva agricultura familiar do município de Marapanim, com ênfase na produção de alimentos para subsidiar - PNAE. Essa estratégia para pesquisa empírica, segundo Yin (2010) deve ser empregada para a investigar "um fenômeno contemporâneo, em seu contexto real, possibilitando a explicação de ligações causais de situações singulares". 
O município é banhando pelo

oceano atlântico e pela Bacia Hidrográfica do Marapanim (Figura 1) que abrange 12

municípios no estado do Pará.

Figura 1. Mapa de localização da área de estudo.

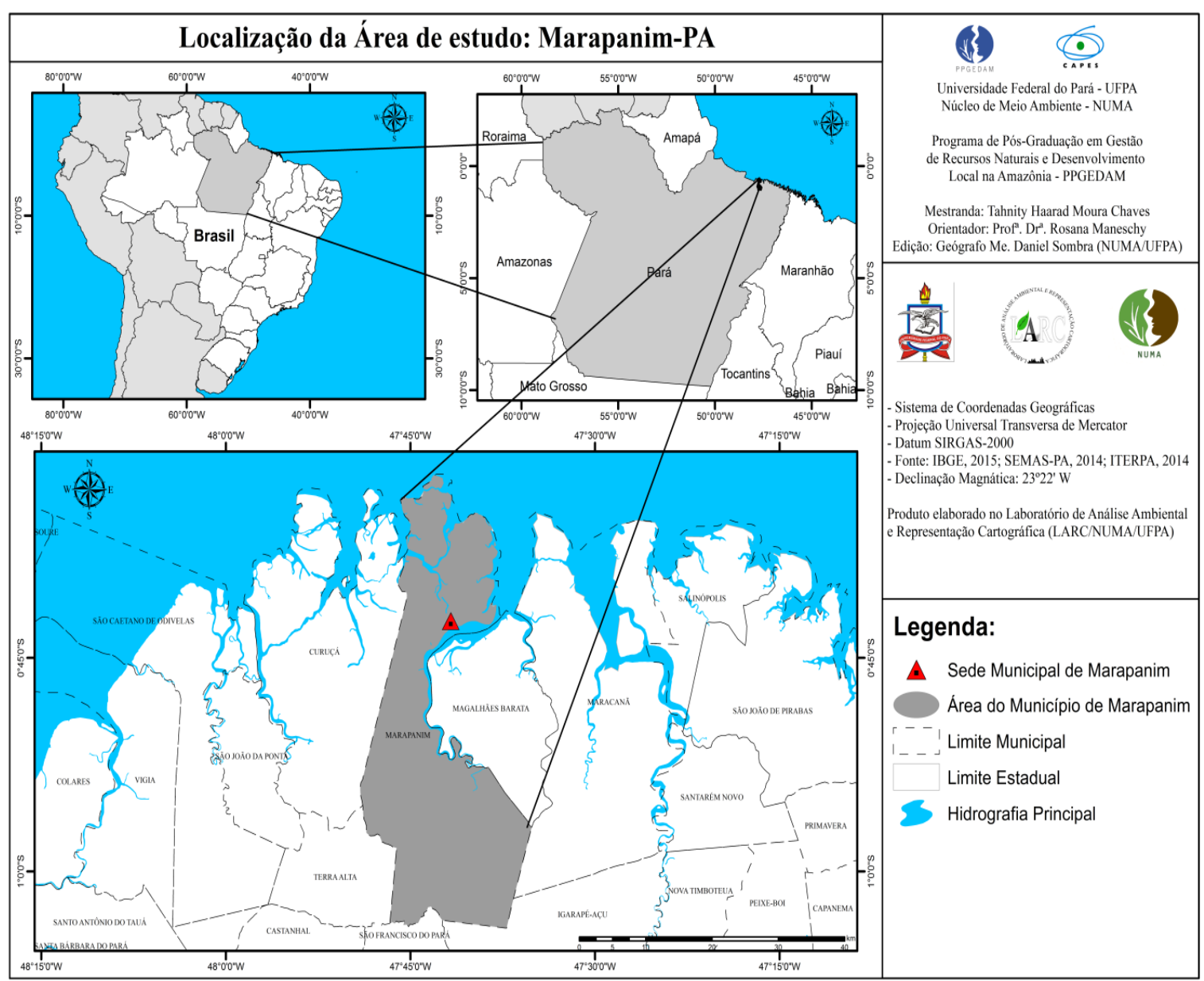

Fonte: Mapa elaborado pelo Laboratório de Análise Ambiental e Representação Cartográfica (LARC/NUMA/UFPA), 2017.

A escolha do caso se deu a partir das ações da Universidade Federal do Pará (UFPA) junto ao grupo "Erva Vida"1 no âmbito do projeto "Rede para o
Fortalecimento e Inovação Estratégica da Cadeia Produtiva de Extratos e Óleos da Amazônia Paraense aplicados a Fitoterápicos e Cosméticos" desenvolvido

\footnotetext{
1 O grupo "Erva Vida" é constituído por mulheres que desenvolvem fitoterápicos há mais de 21 anos e que vem discutindo com a comunidade local a temática do uso das plantas medicinais para
}

produção de fitoterápicos a partir dos saberes de mulheres erveiras-pescadoras, assim como a temática dos "alimentos saudáveis" direcionada principalmente aos jovens da comunidade. 
pelo Núcleo de Meio Ambiente (NUMA)

no município. Essas ações se materializaram em um convênio entre a UFPA e o município. O município é membro integrante juntamente com outros municípios da Bacia hidrográfica do Marapanim, sendo de grande importância para o estado e o NUMA/UFPA faz parte do Comitê Gestor da Bacia.

Foram coletados dados de fontes secundárias e documentais acerca da prática da agricultura no município para relacionar com as informações obtidas a partir de entrevistas com informantes chave (fonte primária), tais como agricultores familiares, gestores municipais e técnicos. Foram realizadas visitas, de agosto de 2016 a maio de 2017, nas atividades desenvolvidas na área em estudo para caraterização dos atores envolvidos na atividade e observação das práticas agrícolas, bem como os produtos produzidos. Na ocasião das visitas, foram realizadas entrevistas com questões

\footnotetext{
${ }^{2} \mathrm{~A}$ ausência desse repasse até o momento em que a pesquisa foi realizada não foi explicado pelo FNDE
}

abertas para colher informações dos sujeitos a partir do seu discurso livre.

De acordo com dados obtidos do Fundo Nacional de Desenvolvimento da Educação (FNDE), o repasse que é destinado ao PNAE para o município de Marapanim é relacionado com a quantidade de alunos matriculados nas escolas, nas creches. Durante o ano de 2016, foi repassado $R \$ 793.296,00$ reais para a conta da Prefeitura Municipal para destinar este recurso para a compra de alimentos para a merenda escolar aos programas de Ensino Fundamental, PréEscola, Educação de Jovens e Adultos (EJA), Ensino Médio, o Mais EducaçãoFundamental e Creche (Tabela 1).

Durante os meses de março, abril e maio de 2017 foi repassado ao município $\mathrm{R} \$ 305.913,00$ reais do FNDE (2017), sendo que no mês de março o programa de ensino Mais Educação-Fundamental não recebeu o repasse ${ }^{2}$ (Tabela 2). Conforme dados obtidos na pesquisa de campo, até o mês de maio de 2017 as escolas e creches do município não haviam sido 
abastecidas com a merenda escolar.

Devido este fato, de 23 a 26 de maio do ano corrente, coordenadores do PNAE estiveram no município para apurar a razão pela qual os estudantes estavam sem acesso a merenda escolar, uma vez que os repasses do governo federal tinham sido executados.

Tabela 1. Repasses do Governo Federal através do Programa Nacional de Alimentação Escolar (PNAE) ao município de Marapanim, 2016.

\begin{tabular}{lrrrrrrrrr}
\hline Programa & Mar & Abr & Mai & Jun & Jul & Ago & Out & Nov & Dez \\
\hline Ensino Fundamental & 32.640, & 32.640, & 32.640, & 32.640, & 32.640, & 32.640, & 32.640, & 32.640, & 32.640, \\
& 00 & 00 & 00 & 0 & 00 & 00 & 00 & 00 & 00 \\
Pré-Escola & $8.370,0$ & $8.370,0$ & $8.370,0$ & $8.370,0$ & $8.370,0$ & $8.370,0$ & $8.370,0$ & $8.370,0$ & $8.370,0$ \\
& 0 & 0 & 0 & 0 & 0 & 0 & 0 & 0 & 0 \\
EJA & $3.618,0$ & $3.618,0$ & $3.618,0$ & $3.618,0$ & $3.618,0$ & $3.618,0$ & $3.618,0$ & $3.618,0$ & $3.618,0$ \\
& 0 & 0 & 0 & 0 & 0 & 0 & 0 & 0 & 0 \\
Alimentação Escolar & 770,00 & 770,00 & 770,00 & 770,00 & 770,00 & 770,00 & 770,00 & 770,00 & 770,00 \\
Ensino Médio & $9.162,0$ & $9.162,0$ & $9.162,0$ & $9.162,0$ & $9.162,0$ & $9.162,0$ & $9.162,0$ & $9.162,0$ & $9.162,0$ \\
& 0 & 0 & 0 & 0 & 0 & 0 & 0 & 0 & 0 \\
Mais Educação & 32.004, & 32.004, & 32.004, & 32.004, & 32.004, & 32.004, & 32.004, & 32.004, & 32.004, \\
Fundamental & 00 & 00 & 00 & 0 & 00 & 00 & 00 & 00 & 0 \\
Creche & $1.580,0$ & $1.580,0$ & $1.580,0$ & $1.580,0$ & $1.580,0$ & $1.580,0$ & $1.580,0$ & $1.580,0$ & $1.580,0$ \\
& 0 & 0 & 0 & 0 & 0 & 0 & 0 & 0 & 0 \\
\hline Total & 88,144, & 88,144, & 88,144, & 88,144, & 88,144, & 88,144, & 88,144, & 88,144, & 88,144, \\
& 00 & 00 & 00 & 00 & 00 & 00 & 00 & 00 & 0 \\
\hline
\end{tabular}

Fonte: Fundo Nacional de Desenvolvimento da Educação - FNDE (2017).

Tabela 2. Repasses do Governo Federal através do Programa Nacional de Alimentação Escolar (PNAE) ao município de Marapanim, 2017.

\begin{tabular}{lrrr}
\hline Programa & Mar & \multicolumn{2}{c}{ Mai } \\
\hline Ensino Fundamental & $37.641,60$ & $37.641,60$ & $37.641,60$ \\
Pré-Escola & $8.077,20$ & $8.077,20$ & $8.077,20$ \\
EJA & $2.912,00$ & $2.912,00$ & $2.912,00$ \\
Alimentação Escolar & 816,20 & 816,20 & 816,20 \\
Ensino Médio & $13.190,40$ & $13.190,40$ & $13.190,40$ \\
Mais Educação - Fundamental & & $54.570,60$ & $54.570,60$ \\
Creche & $2.953,20$ & $2.953,20$ & $2.953,20$ \\
\hline Total & $65.590,60$ & $120.161,20$ & $120.161,20$ \\
\hline
\end{tabular}

Fonte: Fundo Nacional de Desenvolvimento da Educação - FNDE (2017). 
A gestão municipal informou que até maio de 2017 ainda não havia sido empossado um Secretário de Educação. Durante a visita a Secretaria de Agricultura do município, o gestor atual e técnico do órgão, relataram que essa secretaria nunca foi notificada em gestões anteriores apoiar os pregões de compra e elaboração do cardápio da merenda das escolas. Observou-se a necessidade do diálogo entre as instituições para se obter um levantamento da produção local e verificar se o que é produzido no município é capaz de atender a demanda da merenda escolar. Segundo Spinelli e Canesqui (2002) os cardápios devem atender as necessidades nutricionais dos alunos.

A aquisição de alimentos para o PNAE durante as chamadas públicas deve ser de responsabilidade intersetorial, ao qual atuam outros órgãos municipais além da secretaria de educação. Segundo Braga e Azevedo (2012) os sujeitos envolvidos no processo também devem atuar de forma participativa, a saber:

${ }^{3}$ O prefeito da gestão anterior e o secretário de finanças tiveram prisão decretada em 27 de abril de 2017 por crime de responsabilidade e desvio de dinheiro público. A atual gestão encontrou a agricultores, técnicos agropecuários, gestores escolares, merendeiras, nutricionistas, conselho municipal para elaboração de políticas públicas locais e adequação de legislação sanitárias e tributárias. A realidade dos municípios brasileiros é uma falta de gestão participativa entre os órgãos municipais e a descontinuidade do atendimento dos serviços à população ${ }^{3}$.

Verificou-se que as compras têm sido feitas de produtores de outros municípios, por vários entraves existentes em Marapanim. Segundo informações da secretaria de agricultura, Marapanim é o maior produtor de melancia do estado do Pará, porém, isso não é contabilizado para o município (pois o escoamento se faz pelo município de (uruçá) e nem oferecido na merenda escolar.

Existe uma diversificação de alimentos no município, com produtos que reforçam a soberania alimentar, a partir do resgate dos hábitos alimentares locais. As principais culturas agrícolas produzidas pela agricultura familiar em

\footnotetext{
prefeitura com as contas zeradas (http://g1.globo.com/pa/para/noticia/justicadecreta-prisao-de-ex-prefeita-de-marapanimno-pa.ghtml).
} 
Marapanim são: abóbora (Cucurbita spp), açaí (Euterpe oleracea Mart.), acerola (Malpighia glabra L.), arroz (Oryza sativa L.), banana (Musa spp.), batata doce (Ipomoea batatas (L.) Lam.), cana de açúcar (Saccharum officinarum L.), cará (Dioscorea alata L.), coco (Cocos nucifera L.), cupuaçu (Theobroma grandiflorum (Willd. ex Spreng.) Schum.), feijão (Phaseolus vulgaris L.), laranja (Phaseolus vulgaris L.), limão (Citrus limon L.), maçã (Pyrus malus L.), macaxeira/mandioca (Manihot esculenta Crantz.), mamão (Carica papaya L.), maracujá (Passiflora sp.), maxixe (Cucumis anguria L.), melancia (Citrullus lanatus (Thunb.) Matsum.), milho (Zea mays L.), muruci (Byrsonima crassifólia (L.) Rich), pepino (Cucumis sativus L.), pimenta do reino (Piper nigrum L.), pupunha (Bactris gasipaes (Kunth) e tangerina (Citrus reticulata Blanco).

Foi verificado a inserção de culturas atípicas a região, como por exemplo, a maçã e o desenvolvimento da piscicultura em tanques nas propriedades familiares. Os agricultores também têm investido na criação de aves, apicultura e porcinos.
Segundo a gestão municipal, o cultivo de hortaliças também tem potencial para comercialização e fornecimento a merenda escolar porque em geral os agricultores possuem roça, mas cultivam em seus quintais espécies de interesse econômico, tais como: alface (Lactuca sativa L.), cebolinha (Allium fistulosum L.), coentro (Coriandrum sativum L.), couve (Brassica oleracea L.), jambu (Acmella oleracea (L.) R.K.Jansen.), tomate cereja (Solanum lycopersicum var. cerasiforme) e plantas medicinais (Figura 2). Com respeito ao plantio e manejo das hortas, os agricultores utilizam sementeiras; e alguns realizam aplicação de herbicida antes da semeadura. Os mesmos realizam capinas periódicas na área de plantio, adubação verde e efetuam a compostagem a partir de esterco de bovino ou aves que é adquirido no município de Castanhal.

município conta com a presença de um escritório da Empresa de Assistência Técnica e Extensão Rural do Estado do Pará para prestar assistência técnica aos agricultores familiares do município; e os agricultores estão organizados em associações. Todavia, verificou-se que 
apesar de existir produção agrícola diversificada, não existe sistematização de dados da produção a fim de subsidiar as políticas de aquisição de alimentos e orientar a gestão municipal no processo de aquisição. Apesar da agricultura familiar no município estar organizada em associações e cooperativas, o entrave para o fornecimento de alimentos para a merenda escolar tem sido a gestão municipal ineficiente que em função da descontinuidade das ações de apoio à agricultura no município. Assim o PNAE, que segundo Schröde (2010) é um programa que tem incentivado a o desenvolvimento local a partir da compra de alimentos produzidos pela agricultura familiar, não tem logrado sucesso em Marapanim-PA.

Figura 2. Horta em quintal de agricultor familiar, Marapanim-Pará.
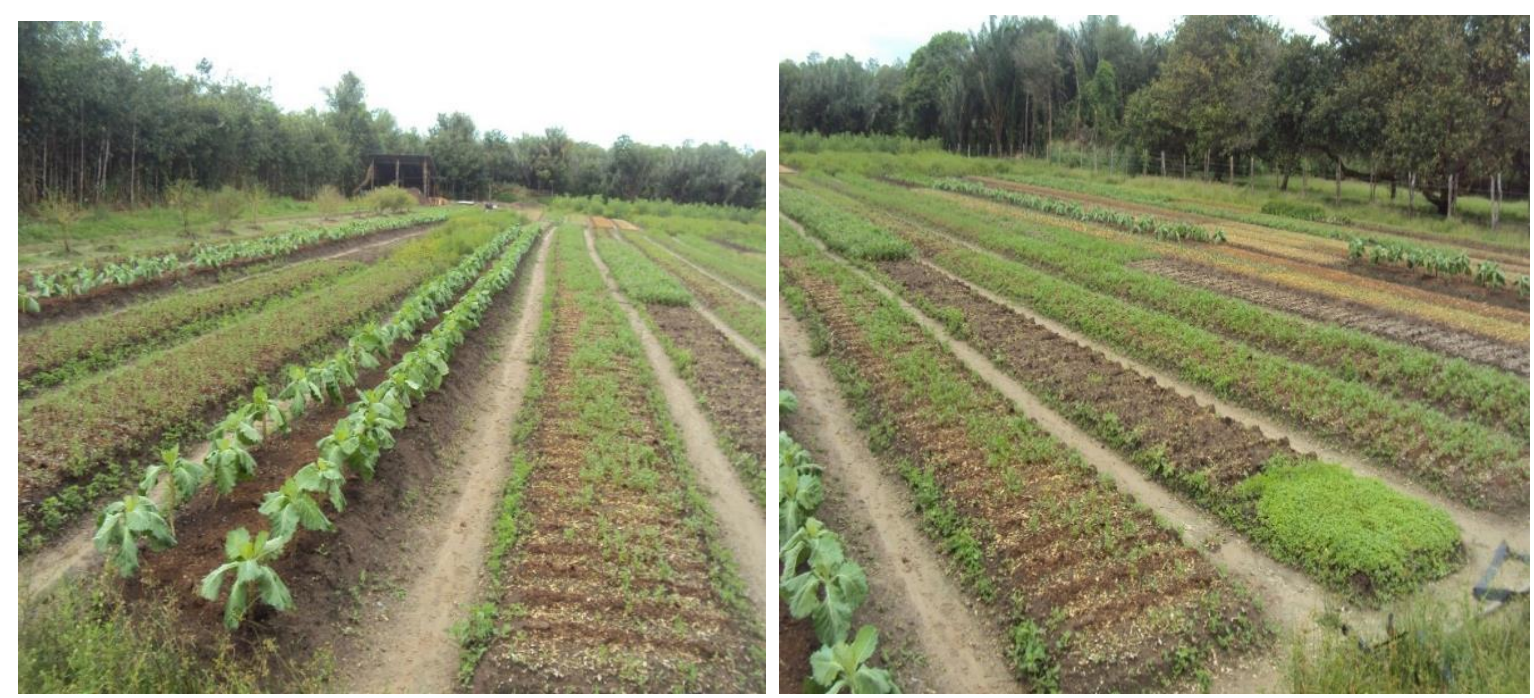

Fonte: Registro da autora, 2017.

Os gestores municipais atuais estão comprometidos em levantar e sistematizar as informações sobre a atividade agropecuária do município, apoiar a agricultura familiar local, e de buscar capacitação ao corpo técnico das secretarias de agricultura e educação, bem como das escolas a fim de apoiar a efetivação do PNAE no município.

A pesquisa apontou como principais entraves na aquisição de alimentos para alimentação escolar via PNAE no 
Município de Marapanim - PA a ausência de uma política intersetorial e descontinuidade nas ações da gestão municipal. Não existem registros das informações agropecuárias atualizadas no município que possam orientar as ações dos gestores e essas ações não estão envolvendo de forma participativa os sujeitos interessados na política de aquisição de alimentos no município.

\section{AGRADECIMENTOS}

Ao Programa de Pós-Graduação em Gestão de Recursos Naturais e Desenvolvimento Local na Amazônia do NUMA/UFPA.

\section{REFERÊNCIAS}

BRAGA, E. M. F.; AZEVEDO, H. S. Segurança alimentar e nutricional: os desafios da intersetorialidade. Revista Aval, v. 2, n. 10, p. 57-71, Jul/Dez, 2012.

GAVIOLI, F. R. Multifuncionalidade da Agricultura e Território: notas a partir de um estudo no assentamento Monte Alegre. Campo-Território, v. 6, n. 11, 2011. Disponível em: <Http://www.seer.ufu.br/index.php/camp oterritorio/article/view/12069>. Acesso em: 25 abr 2017.

HONDA, Y. F.; GOMES, S. C.; CABRAL, E. R. Participação dos produtores familiares no PAA: estratégias de produção e comercialização em área periurbana do município de Ananindeua, PA. Revista Cesumar Ciências Humanas e Sociais Aplicadas, v.21, n.1, p. 125-145, jan./jun. 2016.

MDSA, Ministério do Desenvolvimento Social e Agrário. Diálogo entre povos da Amazônia combate insegurança alimentar na região. 2017. Disponível em: <http://www4. planalto.gov.br/consea/co municacao/noticias/2017/dialogo-entrepovos-da-amazonia-vai-combaterinseguranca-alimentar-e-ajustarpoliticas-publicas-a-regiao/view>.

SANTANA, A. C. de; SEQUEIRA, G. R.; OLIVEIRA, C. M. de; GOMES, S. C. Mercado institucional e agricultura urbana e periurbana em Curuçambá, Ananindeua, Pará: Oportunidades e desafios. G\&DR, v. 13, n. 1, p. 316-338, janabr/2017.

SCHRÖDE, M. Desenvolvimento rural, meio ambiente e políticas públicas: os caminhos do fortalecimento da agricultura familiar na Amazônia brasileira. PRACS, n. 3, p. 53-69, dez. 2010.

YIN, R. K. Estudo de Caso: Planejamento e Métodos. $4^{a}$ Ed. São Paulo: BOOKMAN, 2010. 212 p.

SPINELLI, M. A. dos S.; CANESQUI, A. M. O programa de alimentação escolar no estado de Mato Grosso: da centralização à descentralização (1979 - 1995). Rev.

Nutr. v.15, n.1Campinas Jan. 2002. 